\title{
Assessment of quality of nutrition information materials for pelvic cancer patients in the UK
}

\author{
G. Saltaouras ${ }^{1,2}$, H. Lightowler ${ }^{1}, \mathrm{~S} . \mathrm{Coe}^{1}, \mathrm{~J}$. Brett ${ }^{2}$ and E. Watson ${ }^{2}$ \\ ${ }^{1}$ Oxford Brookes Centre for Nutrition and Health, Department of Sport, Health Sciences \& Social Work, Oxford \\ Brookes University, Gipsy Lane, OX3 OBP and \\ ${ }^{2}$ Department of Nursing, Oxford Brookes University, Jack Straw's Lane, OX3 OFL, UK.
}

A cancer diagnosis serves as a 'teachable moment' for many patients to consider health behaviours, such as diet ${ }^{(1)}$. Patients diagnosed with a pelvic malignancy (prostate, bladder, rectum or gynaecology) may be interested in changing their dietary habits ${ }^{(2)}$. Improved dietary habits may reduce the risk of disease progression in these cancer groups ${ }^{(3)}$. However, lack of access to information from healthcare professionals may prompt them to seek advice from online sources ${ }^{(4)}$. To the authors' knowledge, the quality of patient information from online sources has not been assessed. The aim of the study is to evaluate the content and readability of the available online patient information materials (PIMs) in relation to diet and nutrition specifically for pelvic cancer patients.

PIMs relating to diet and nutrition following a cancer diagnosis were identified by searching in Public Sector organisations, Cancer Centres and registered charities throughout the UK. Searches included key terms related to cancer in the pelvic area or generic cancer and related to provision of information about a healthy diet after diagnosis, nutritional management of treatment-related symptoms or weight management. Content was assessed with an evidence-based checklist developed by the Picker Institute ${ }^{(5)}$. Readability was assessed with the Flesch Reading Ease score (FRE), Flesch-Kincaid Grade Level (FKGL) and the Simplified Measure of Gobbledygook (SMOG) Grade.

Few cancer centres (5/14) and charities (8/196) had online information on diet and nutrition. A total of 18 PIMs were identified; 17 were leaflets/booklets and one source (Cancer Research UK) had online information only. Four PIMs were pelvic cancer-specific (prostate, bladder and bowel cancer) and 14 were generic cancer. The NHS Choices website had no online information specifically on diet and cancer but provided a link to other sources for information. Content scores ranged from 22-36/40. PIMs from charities scored higher than the ones from cancer centres $(n 11 ; 30.6 \pm 3.7 \mathrm{Vs} n 7 ; 27.0 \pm 4 \cdot 1, P=0.068)$. The average readability level was $8^{\text {th }}$ grade-level, which corresponds to the reading ability of 13-14 year-olds. One third of the materials scored higher than the upper acceptable reading level.

\begin{tabular}{lllllll}
\hline Readability Index & FRE & SD & FKGL & SD & SMOG & SD \\
\hline Mean US Grade Reading Level & $8 \cdot 2$ & $1 \cdot 3$ & $8 \cdot 3$ & $1 \cdot 6$ & $7 \cdot 8$ & $1 \cdot 0$ \\
Age equivalent (years) & $13-14$ & & $13-14$ & & $13-14$ & \\
PIMs above $8^{\text {th }}$ grade level (\%) & $33 \%$ & & $39 \%$ & & $33 \%$ & \\
Grade Level Range & $7-12$ & & $6-12$ & & $6-10$ & \\
\hline
\end{tabular}

This study highlights the lack of targeted, reliable and easy to understand patient information about diet and nutrition in pelvic cancer. The content and the language of some of the current materials may need to be revised.

1. Van Mossel C, Leitz L, Scott S et al. (2012) Eur J Cancer Care (Engl) 21, 296-320.

2. Avery KNL, Donovan JL, Gilbert R et al. (2013) Cancer Causes Control 24, 1119-1128.

3. Richman EL, Carroll PR \& Chan JM (2012) Int J Cancer 131, 201-210.

4. Rozmovits L and Ziebland S (2004) Patient Educ Couns 53, 57-64.

5. Coulter A, Ellins J, Swain D et al. (2006) Picker Institute Europe. 